\title{
Les enfants réfugiés, la déscolarisation et les technologies mobiles
}

Université de Montréal

Université de Genève

Des agences internationales comme l'UNICEF et le gouvernement de certains pays comme la Norvège sont très impliqués dans des initiatives visant le développement d'outils d'apprentissages mobiles pour les enfants réfugiés. EduApp4Syria est par exemple une application éducative créée expressément pour les réfugiés syriens. Malgré l'intérêt de ces projets pour permettre l'accès à l'éducation dans des contextes fragiles, nous nous interrogeons sur les enjeux éducatifs, sociaux ou économiques sous-jacents.

En 2015, le nombre de personnes déplacées de force dans le monde a atteint son plus haut taux depuis la fin de la Deuxième Guerre mondiale (Haut Commissariat des Nations unies pour les réfugiés, 2015). Des générations d'enfants réfugiés originaires d'Afghanistan ou de Syrie ont dû abandonner leur maison et interrompre leur formation. Bien que l'afflux de réfugiés en Europe ait joui d'une forte visibilité médiatique, $86 \%$ des réfugiés sont accueillis dans des pays en développement (UNESCO, 2014). Les communautés hôtes sont submergées par cet afflux et sont incapables de répondre à la demande pour l'accès aux services de base tels que l'éducation.

\section{L'éducation n'est pas garantie}

Les pays d'accueil ont souvent un système d'éducation fragile et une infrastructure inadéquate pour accueillir un surplus important d'enfants. D'ailleurs, dans les camps pour réfugiés, rares sont les enseignants qualifiés.

Ainsi, seulement $50 \%$ des enfants réfugiés suivent un enseignement primaire (Haut Commissariat des Nations unies pour les réfugiés, 
2015). Le risque de non-scolarisation est cinq fois plus élevé pour les enfants réfugiés que pour les autres enfants. Pourtant, l'éducation est un droit universel et un élément essentiel du développement économique et social des nations.

Pour pallier cette situation, des organisations comme le Haut Commissariat des Nations unies pour les réfugiés (UNHCR) ou l'UNICEF recourent aux technologies mobiles pour délivrer des contenus éducatifs. Effectivement, dans les communautés marginalisées qui nont pas accès à Internet ou à des ordinateurs, l'utilisation du téléphone mobile est répandue et elle permet une meilleure équité dans la distribution des connaissances (UNESCO, 2012).

\section{Quelques projets éducatifs employant les technologies mobiles}

Le projet EduApp4Syria a été lancé en 2015 par le ministère des Affaires étrangères de la Norvège et ses partenaires qui ont développé deux jeux pour apprendre l'arabe en utilisant le cellulaire.

Un de ces jeux, Sima, est basé sur le plan d'étude syrien. Il a été validé par des experts en alphabétisation et des psychologues cognitivistes. Il se démarque par la qualité de ses contenus. La narration s'appuie sur la culture syrienne, ce qui facilite l'identification des enfants aux personnages et à l'histoire. La qualité de ce jeu est à souligner dans un contexte où souvent le matériel de formation développé dans les pays du Nord est peu adapté au contexte des pays du Sud (Dzakiria, Razak et Mohamed, 2004). Ce problème est encore plus marqué dans le cas des technologies mobiles, car le contenu n'est pas toujours développé par des éducateurs et la qualité est souvent insatisfaisante (UNESCO, 2011).

Un autre projet destiné aux réfugiés syriens dans un camp au Liban est « Raspberry Pi ». L'UNICEF et les entreprises partenaires ont voulu développer un petit ordinateur peu dispendieux permettant d'accéder à des contenus éducatifs. C'est ainsi quen 2015 au Liban un centre d'apprentissage informel a été ouvert pour les enfants réfugiés syriens âgés de 10 à 16 ans où ils peuvent suivre des cours en arabe de Khan Academy grâce à Raspberry Pi. De plus, cet outil offre la possibilité d'apprendre à coder en utilisant le langage Scratch.

\section{La nécessité de mener des études scientifiques}

Bien que de nombreuses recherches aient été menées sur des projets éducatifs impliquant les technologies, peu d'attention a été portée à ceux implantés dans les communautés défavorisées des réfugiés vivant dans des enclaves isolées et temporaires (Yerousis et al., 2015). Les écrits sur ces projets ont principalement été publiés par les organisations internationales ou leurs partenaires à l'origine de ces initiatives. Cela pose problème quant à l'objectivité des idées véhiculées et les avantages réels de ces outils.

En effet, malgré la plus-value de ces initiatives qui permettent l'accès autrement très difficile ou impossible à des contenus éducatifs, le rôle des enseignants et leur accompagnement des enfants dans l'apprentissage par le biais de technologies mobiles ne sont pas clairement définis.

Or, comme l'UNESCO le précise dans ses Principes directeurs pour l'apprentissage mobile, le potentiel est dans les usages, car « la technologie mobile n'est pas et ne sera jamais une panacée éducative. Mais 
elle est un outil puissant et encore trop souvent ignoré (...).» (2013, p. 8). Le rôle des enseignants et leur préparation pour intégrer efficacement les technologies dans leur enseignement sont donc fondamentaux.

\section{Conclusion}

À ce jour, il est difficile de savoir comment des enseignants peu qualifiés et travaillant dans des camps où le niveau de rotation est élevé arrivent à intégrer les technologies dans leur enseignement, car les conditions d'enseignement sont très difficiles (Haut Commissariat des Nations unies pour les réfugiés, 2015). Nous nous demandons aussi quelle est la durabilité de projets nés comme une réponse à une urgence. Les enjeux nous semblent cruciaux, tant sur le plan de la progression des apprentissages des enfants qu'au niveau des ressources économiques déployées. Nous ne pouvons donc souhaiter que davantage d'études empiriques soient menées afin que le potentiel des TIC soit exploité au mieux au bénéfice des apprenants et pour l'avancement des connaissances scientifiques dans ce domaine.

\section{Références}

Dzakiria, H., Razak, A. A. et Mohamed, A. H. (2004). Improving distance courses: Understanding teacher trainees and their learning styles for the design of teacher training courses and materials at a distance. The Turkish Online Journal of Distance Education, 5(1), 1-18. Repéré à http://tojde.anadolu.edu.tr/yonetim/icerik/makaleler/118-published.pdf

Haut Commissariat des Nations unies pour les réfugiés. (2015). UNHCR global trends: Forced displacement in 2014. Repéré à http://unhcr.org/556725e69.html

UNESCO. (2011). UNESCO mobile learning week report. Repéré à http://www.unesco.org/fileadmin/MULTIMEDIA/ HQ/ED/ICT/pdf/UNESCO\%20MLW\%20report\%20final\%2019jan.pdf

UNESCO. (2012). Mobile learning for teachers. Global themes. Repéré à http://unesdoc.unesco.org/images/0021/002164/216452E.pdf

UNESCO. (2013). Principes directeurs pour l'apprentissage mobile. Repéré à http://unesdoc.unesco.org/images/0021/002196/219661f.pdf

UNESCO. (2014). Mobile learning for teacher training and curriculum development. Prospects, 44(1), 1-136.

Yerousis, G., Aal, K., von Rekowski, T., Randall, D. W., Rohde, M. et Wulf, V. (2015). Computer-enabled project spaces: Connecting with Palestinian refugees across camp boundaries. Dans B. Begole et J. Kim (dir.), Proceedings of the $33^{\text {rd }}$ Annual ACM Conference on human factors in computing systems (p. 3749-3758). New York, NY : ACM. http://dx.doi.org/10.1145/2702123.2702283

\section{Pour citer cet article}

Caneva, C. (2018). Les enfants réfugiés, la déscolarisation et les technologies mobiles. Formation et Profession, 26(1), 146-148. http://dx.doi.org/10.18162/fp.2018.a143 